\title{
A INFLUÊNCIA DA INOVAÇÃO SUSTENTÁVEL E DO LUXO SOBRE A DISPOSIÇÃO A PAGAR E A INTENÇÃO DE COMPRA DO CONSUMIDOR
}

\author{
Mateus Luan Dellarmelin* \\ mateusluand@gmail.com \\ Eliana Andréa Severo \\ elianasevero2@hotmail.com \\ Josieli Lazzarotto \\ josi.lazzarotto@gmail.com \\ *Faculdade Meridional - IMED
}

http://dx.doi.org/10.1590/1413.2311.058.62218

Recebido em 15/02/2016

Aprovado em 23/06/2017

Disponibilizado em 07/08/2017

Avaliado pelo sistema "double blind review"

Revista Eletrônica de Administração

Editoras-chefe: Andrea Oltramari e Maria Ceci Misoczky

ISSN 1413-2311 (versão "on line")

Editada pela Escola de Administração da Universidade Federal do Rio Grande do Sul

Periodicidade: Quadrimestral

Sistema requerido: Adobe Acrobat Reader

\section{RESUMO}

Diante de um contexto de crescentes preocupações relacionadas aos problemas ambientais, as inovações sustentáveis de produto desempenham um papel importante na redução dos impactos ambientais ocasionados pelo consumo. Contudo, o sucesso dessas inovações depende da sua adoção por parte dos consumidores. Por este motivo, o objetivo deste artigo é verificar a influência da inovação sustentável e do luxo sobre a disposição a pagar e a intenção de compra do consumidor. Neste sentido, foi realizadauma pesquisa causal, para verificar as relações de causa e efeito entre variáveis, em dois estudos.O primeiro estudo realizado foi um experimento de um fator, entre-sujeitos, onde a variável independente (sustentabilidade ambiental) foi manipulada. Os resultados do primeiro estudo demonstraram que a informação sobre a sustentabilidade ambiental de uma inovação não influenciou significativamente a intenção de compra do consumidor e a sua disposição a pagar. No segundo estudo realizado, além da sustentabilidade, foi manipulada também a presença da variável luxo. Os resultados do segundo estudo demonstram que a associação da sustentabilidade ao luxonão torna o seu efeito significante, pois o efeito da interação entre as duas variáveis independentes (sustentabilidade e luxo), bem como o efeito principal da sustentabilidade, sobre a disposição a pagar e intenção de compra do consumidor não foi significante. O luxo, por sua vez, teve um efeito negativo sobre a intenção de compra, mas positivo sobre a disposição a pagar.

Palavras-chave: Inovação Sustentável. Disposição a pagar. Intenção de Compra.

\begin{abstract}
In front of a scenario of growing concerns related to environmental issues, product sustainable innovations play an important role in reducing environmental impacts caused by
\end{abstract}


consumption. However, the success of these innovations depends on its adoption by consumers. Therefore, the purpose of this article is to verify the influence of sustainable innovation and luxury on consumer willingness to pay and purchase intent. In this sense, a causal research, to verify cause-and-effect relationships between variables, in two studies. The first study conducted was a one-factor between-subjects experiment, where the independent variable (environmental sustainability) was manipulated. First study results showed that information on environmental sustainability did not influence consumer purchase intent and willingness to pay significantly. In the second study, in addiction to sustainability, it was also manipulated the presence of luxury. In the second study, beyond sustainability, was manipulated the presence also of the luxury. The results of the second study demonstrated that the Association of sustainability the luxury doesn't make your significant effect, because the effect of the interaction between the two independent variables (sustainability and luxury), as well as the main effect of sustainability, on the willingness to pay and consumer purchase intent was not significant. Luxury, in turn, had a negative effect on purchase intent, but a positive effect on willingness to pay.

Keywords: Sustainable innovation. Willingness to Pay. Purchaseintent.

\section{RESUMEN}

En un contexto de creciente preocupación ambiental relacionada, innovaciones de producto sostenible juegan un papel importante en la reducción de los impactos ambientales generados por el consumo. Sin embargo, el éxito de estas innovaciones depende de su adopción por los consumidores. Por esta razón, el objetivo de este artículo es comprobar la influencia de la innovación sostenible y de lujo en la disposición a pagar y la intención de compra del consumidor. En este sentido, una investigación causal, para verificar las relaciones de causa y efecto entre variables, en dos estudios.El primer estudio fue un experimento de un factor, entre-sujeitos, donde se manipuló la variable independiente (sostenibilidad ambiental). Los resultados del primer estudio demostraron que información sobre la sostenibilidad de una innovación no influyó significativamente la intención de compra del consumidor y su disposición a pagar. En el segundo estudio, más allá de la sostenibilidad, fue manipulada la presencia también de lujo. Los resultados del segundo estudio demostraron que la Asociación de la sostenibilidad el lujo no hace su efecto significativo, ya que el efecto de la interacción entre las dos variables independientes (sostenibilidad y lujo), así como el efecto principal de la sostenibilidad, en la disposición a pagar y la intención de compra del consumidor no fue significativo. El lujo, a su vez, tuvo un efecto negativo en la intención de compra, pero positivo sobre la voluntad de pagar.

Palabras Clave: Innovación Sostenible. Disposición a pagar. Intención de compra.

\section{INTRODUÇÃO}

É crescente a preocupação com o meio ambiente e as discussões sobre os problemas ambientais. Com isso, torna-se relevante, também, o desenvolvimento de pesquisas com abordagens sobre inovações sustentáveis como uma forma de consumir causando menos impactos ambientais. Entretanto, a preocupação com os problemas ambientais, por parte dos consumidores, não necessariamente determina o consumo de produtos ecologicamente 
corretos e uma disposição a pagar mais por determinados produtos. Contudo, assim como qualquer inovação de produto, o sucesso de uma inovação ambientalmente sustentável depende da adoção dos consumidores (NOPPERS et al., 2014).

Alguns autores dedicam-se ao estudo de fatores que influenciam os consumidores a adotarem inovações ambientalmente sustentáveis. Claudy, Peterson e O’Driscoll(2013) e Claudy, Garcia e O'Driscoll(2015), por exemplo, argumentam que a adoção de inovações sustentáveis, como fontes de energia renovável, é influenciada tanto por atitudes positivas quanto por atitudes negativas em relação às inovações. As atitudes, por sua vez, segundo os autores, são formadas pelas crenças pré-existentes e por razões contrárias e favoráveis à adoção das inovações sustentáveis.

Os produtos ambientalmente sustentáveis podem ter avaliações mais negativas em relação aos seus atributos funcionais - como preço ou conveniência - se comparados às alternativas tradicionais. Noppers et al. (2014)destacam que apesar destas avaliações negativas influenciarem a adoção de inovações sustentáveis, a presença de atributos ambientais e simbólicos, podeminfluenciar positivamente a adoção de tais inovações.

Nas pesquisas que analisam a adoção de inovações sustentáveis no contexto de bens de alto valor agregado (CLAUDY; PETERSON; O'DRISCOLL, 2013; NOPPERS et al., 2014; CLAUDY; GARCIA; O’DRISCOLL, 2015), poucas exploram a inovação sustentável no contexto de bens de consumo, em específico, dos produtos sustentáveis capazes de transmitir valores simbólicos aliadosao luxo (TYNAN; MCKECHNIE; CHUON, 2010).

As abordagens referentes aos produtos de luxo em sua maioria, detêm-se aos atributos de qualidade percebida, da notoriedade da marca, da distribuição seletiva e refinada, nos preços elevados e sob o ponto de vista do comportamento dos consumidores, as abordagens referem-se às simbologias inerentes ao consumo de produtos de luxo, e à construção da identidade (BEVERLAND, 2004; VIGNERON; JOHNSON, 2004).

Perante o exposto, o objetivo dessa pesquisa é verificar a influência da inovação sustentável e do luxo sobre a intenção de compra e disposição a pagar do consumidor. Para tanto, além desta introdução, na sequência deste artigo são apresentados: i) referencial teórico, elencando a inovação sustentável de produto e produto verde, e produtos de luxo; ii)metodologia da pesquisa; iii) resultados e discussões de dois estudos experimentais; e, iv) considerações finais. 


\section{REFERENCIAL TEÓRICO}

\subsection{Inovação Sustentável de Produto e Produto Verde}

Conforme Almeida (2007), as organizações enfrentam dois desafios ao adotarem práticasdo desenvolvimento sustentável. O primeiro desafio é relacionado à geração de inovações necessárias à existência humana sustentável, ou seja, disponibilizando soluções capazes de desempenhar múltiplas funções.O segundo desafio refere-se a vencer as resistências da sociedade quanto aos novos produtos e serviços, frente às evidências de insustentabilidade dos sistemas produtivos e das atuais soluções tecnológicas existentes no mercado.

As organizações, ao adotarem a prática da inovação sustentável como um mecanismo que permite abranger questões de sustentabilidade, obtêm a possibilidade de conquistar novos segmentos de mercados e clientes, gerando, por meio da própria inovação, um valor agregado e positivo ao capital da firma (HANSEN; GROSSE-DUNKER; REICHWALD, 2009). Neste cenário, identificar os desafios que as empresas terão de superar para alcançar o sucesso comercial dos produtos ecologicamente corretos é essencial para a obtenção deste êxito (DANGELICO, 2010).

No que tange aos produtos verdes, estes são caracterizados como aqueles que possuem as mesmas funções básicas dos produtos convencionais, mas a diferença está em seu impacto sobre o ciclo de vida que é mitigado, trazendo benefícios para todos os usuários envolvidos, sendo estes, o meio ambiente, a empresa e a sociedade (JANSEN; STEVELS, 2006; MICHAUD; LLERENA, 2011). Neste sentido, Lages e Vargas Neto (2002) corroboram afirmando que os produtos verdes não agridem o meio ambiente e a saúde humana, pois os processos e as atividades necessárias para tornar este produto disponível aos consumidores são ambientalmente favoráveis. Além disso, os produtos verdes devem após sua vida útil, ser facilmente reparados, reutilizados, reciclados ou se degradarem rapidamente em contato com o meio ambiente (D`SOUZA; TAGHIAN; KHOSLA, 2007).

Portanto, é importante destacar que a eco-inovação pode ser compreendida como a inovação que reduz os impactos negativos causados pela produção e pelo desenfreado consumo da sociedade, e esta inovação sustentável, pode ser desenvolvida tanto por fabricantes quanto por clientes (KLIMOVA; ZITEK, 2011).

Contudo, a tendência de mercado é que os consumidores, gradativamente, aumentem a sua consciência ambiental, gerando, assim, mais responsabilidade em relação às questões ambientais e atitudes mais favoráveis em relação aos produtos verdes e sustentáveis 
(BLOEMER; RUYTER, 2002). Diante do exposto, elenca-se a primeira hipótese deste estudo:

Hipótese 1: A inovação sustentável influencia positivamente (H1a) a intenção de compra do consumidor e (H1b) a disposição a pagar.

\subsection{Produtos de Luxo}

As principais características dos produtos de luxo são a elevada qualidade do material, altos preços, o consumo associado ao poder, a exclusividade e o consumo conspícuo (FIONDA; MOORE, 2009; HEINE; PHAN, 2011; JIN, 2012), associados a um consumo simbólico, as marcas de luxo, controlam a distribuição dos produtos, mantendo sua base de consumidores e aumentando o preço médio (BRUN; CASTELLI, 2013; KAPFERER; FLORENCE, 2016).

Estas características dos produtos de luxo podem ser subdivididas em expertise e competência técnica do fabricante. Derivando de sua experiência ou de um foco de pesquisa e desenvolvimento(P\&D) e inovação, a complexidade da manufatura, dos materiais e componentes, da construção e do design funcional do produto, do trabalho artesanal perfeito, dos atributos do produto, serviços agregados, conforto e usabilidade, valor e durabilidade, funcionalidade e desempenho (HEINE; PHAN, 2011).

Para corroborar com as características intangíveis dos produtos de luxo, Scarabotto et al. (2006), discorrem que consumir os produtos de luxo é uma experiência que envolve todos os sentidos, ativando as emoções e as fantasias. Neste sentido, Tynan, McKechnie e Chuon (2010) afirmam que os produtos de luxo são considerados aqueles que oferecem altos níveis de valores simbólicos e hedônicosvinculados às experiências dos consumidores.

Algumas pesquisas indicam que os consumidores de produtos de luxo estão dispostos a pagar um preço mais elevado por este tipo de produto, resultante do valor percebido do prazer hedônico e da percepção utilitária realizada acerca destes (BODUR; GAO; GROHMANN, 2014; PATRICK; PROKOPEC, 2015).

Diante do exposto, espera-se que os consumidores estejam dispostos a pagar mais por inovações de produtos que sejam ligados à marcas de luxo. Contudo, mesmo que os produtos de luxo estejam associados a valores hedônicos (TYNAN; MCKECHNIE; CHUON, 2010) e que a percepção de qualidade associada ao luxo impulsiona a decisão de compra (FIONDA; MOORE, 2009; HAGTVEDT; PATRICK, 2009), a consciência de que estes produtos têm um 
preço mais elevado, pode fazer com que a intenção de compra seja menor. Desta forma propõe-se a segunda hipótese desse estudo:

Hipótese 2: O efeito da informação da sustentabilidade do produto de luxo exerce (H2a) um efeito negativo sobre intenção de compra do consumidor e (H2b) efeito positivo sobre a disposição a pagar.

\section{METODOLOGIA DA PESQUISA}

\subsection{Estudo 1}

Considerando o objetivo desse artigo, que é verificar a influência da inovação sustentável sobre a intenção de compra, optou-se por realizar uma pesquisa causal, que permite verificar as relações de causa e efeito entre variáveis. Como primeiro estudo, foi realizado um experimento de um fator, entre-sujeitos, onde a variável independente sustentabilidade ambiental - foi manipulada, a fim te testar as hipóteses H1a e H1b.

No início do questionário era solicitado aos participantes que, ao acessarem um site de negócios, iriam se deparar com uma notícia a respeito de uma marca de bolsas e mochilas que estava lançando uma nova linha de produtos. Este questionário possuía 12 questões, as quais foram validadas por um Doutor expert na área temática de estudo. Coerentemente, foi impresso o questionário assim como a descrição do cenário 1, excepcionalmente para a pesquisa, sendo respondido individualmente pelos participantes. Na condição de inovação sem a informação de sustentabilidade ambiental (grupo de controle), os participantes realizaram a leitura da seguinte situação do cenário 1 :

A marca Unwind está lançando no mercado uma nova linha de mochilas e bolsas com diversos estilos e cores. Para a composição do produto, a marca utiliza tecidos de poliéster, nylon e algodão, entre outros materiais. As mochilas e bolsas da Unwind são vendidas exclusivamente no site da empresa e entregues para toda as regiões do País.

Já na condição de inovação com informação de sustentabilidade, os participantesse depararam com a seguinte situação:

A marca Unwind está lançando no mercado uma nova linha de mochilas e bolsas com diversos estilos e cores. Para a composição do produto, a marca utilizamatérias-primas 
recicladas como tecidos de guarda-chuvas e as câmaras de pneus. As mochilas e bolsas da Unwind são vendidas exclusivamente no site da empresa e entregues para toda as regiões do País.

\subsubsection{Mensurações}

Para a verificação de manipulação foi utilizado um item, medido em uma escala Likertde 7 pontos (Discordo totalmente/Concordo totalmente), onde o respondente deveria indicar o grau de concordância com a afirmação de que a situação lida referia-se a uma linha de produtos ecologicamente corretos.

A intenção de compra foi mensurada com um item, medido em uma escala Likertde 7 pontos (Qual a probabilidade de você comprar uma bolsa ou mochila da nova linha lançada pela Unwind? Muito improvável/Muito provável). Já a disposição a pagar foi verificada por meio de uma pergunta aberta, onde o respondente deveria escrever o quanto estaria disposto a pagar, em reais, por um produto da linha descrita na situação (HOMBURG; KOSCHATE; HOYER, 2005).

Como variável de controle, foi mensurada a consciência ambiental a partir da dimensão do comportamento em relação aos produtos, da escala desenvolvida por Straughan e Roberts (1999). Esta variável, no entanto, não teve efeito de controle significante tantosobre intenção de compra $(\mathrm{F}(1,61)=0,151 ; \mathrm{p}=0,699)$, quanto sobre disposição a pagar $(\mathrm{F}(1$, 61)=0,008; $\mathrm{p}=0,931$ ) e por isso não foi considerada nas análises finais. $\mathrm{O}$ efeito de controle da renda sobre a disposição a pagar também foi verificado, sendo este significante $(\mathrm{F}(1$, $61)=7,801 ; p<0,01$ ) e por este motivo renda foi incluída como uma covariável.

No que se refere à amostra, participaram do estudo 70 estudantes de cursos de graduação e pós-graduação em nível de especialização de uma faculdade do Norte do Rio Grande do Sul, distribuídos aleatoriamente entre os dois grupos experimentais. Foram excluídos 6 participantes, pois apresentavam mais de dois desvios padrões da média de pelo menos uma das variáveis dependentes. Portanto, a amostra final foi composta por 64 participantes, sendo $76 \%$ do gênero feminino. A média de idade foi de 29,13 anos, sendo a idade mínima de 20 anos e a máxima de 44.

\subsection{Estudo 2}

O estudo 2 teve um desenho experimental 2 (informação de sustentabilidade: com; sem) x 2 (luxo: com; sem), entre sujeitos, a fim de testar as hipóteses H2a e H2b. O estudo 
teve como base o mesmo contexto do estudo anterior e a informação de sustentabilidade foi manipulada da mesma forma. O questionário possuía 12 questões, as quais também foram validadas por um Doutor expert na área temática de estudo. Consoante a isso, foi impresso o questionário bem como a descrição do cenário 2, excepcionalmente para a pesquisa, sendo respondido individualmente pelos participantes. Para a manipulação da variável luxo, foi acrescentada, no início do cenário 2 , a informação de que a marca era considerada uma marca de luxo. (A marca de luxo Unwind está lançando no mercado uma nova linha de mochilas e bolsas com diversos estilos e cores.).

A verificaçãode manipulação para o fator sustentabilidade, consciência ambiental e variáveis demográficas foram mensuradas da mesma forma que no estudo 1. Para a verificaçãode manipulação para a variável luxo, foi solicitado aos participantes que indicassem, de 1 a 7, o grau de concordância com a afirmação de que a situação se referia a uma linha de produtos de luxo.

Como variáveis de controle, foram mensuradas a consciência ambiental da mesma forma que no estudo 1 e o consumo por status, com 5 itens retirados da Escala de Consumo de Status elaborada por Eastman, Goldsmith e Flynn (1999), traduzida e adaptada no Brasil por Strehlau e Aranha (2004).A consciência ambiental não teve efeito tanto sobre intenção de compra $(\mathrm{F}(1,102)=1,900 ; \mathrm{p}=0,171)$ quanto sobre disposição a pagar $(\mathrm{F}(1,102)=0,244$; $\mathrm{p}=0,623$ ), por esse motivo não foi considerada para as análises finais. Já consumo por status não teve efeito sobre intenção de compra $(\mathrm{F}(1,102)=0,582 ; \mathrm{p}=0,447)$, mas teve sobre disposição a pagar $(\mathrm{F}(1,99)=3,920 ; \mathrm{p}<0,05)$, sendo incluída como covariável nesta análise. Além disso, também foi considerada o efeito da renda do participante sobre a disposição a pagar, sendo esse efeito significante $(\mathrm{F}(1,99)=12,680 ; \mathrm{p}<0,001)$ e, portanto, incluído como covariável na análise.

Participaram do estudo 116 consumidores de uma cidade do norte do Rio Grande do Sul, distribuídos aleatoriamente entre os 4 grupos experimentais. Foram excluídos9participantes das análises, por estarem acima de dois desvios padrões da média de pelo menos uma das variáveis dependentes. A amostra final ficou composta por 108 participantes, sendo $65 \%$ do sexo feminino. A média de idade dos participantes do estudo 2 foi de 30,61 anos, sendo a idade mínima de 14 anos (para apenas um dos participantes, o qual não foi considerado na análise final) e a máxima de 68 .

\section{RESULTADOS E DISCUSSÕES}

\subsection{ESTUDO 1}

REAd | Porto Alegre - Edição 86 - No 2 - Maio / Agosto 2017 - p. 258 - 273 


\subsubsection{Verificaçãode manipulação}

Os resultados da verificaçãode manipulação demonstraram que a manipulação foi bem sucedida, ou seja, na condição com sustentabilidade, participantes perceberam que se tratava de uma linha de produtos ecologicamente corretos $(M=5,36)$, enquanto na condição sem sustentabilidade, os participantes não tiveram essa percepção $(M=2,84 ; F(1,62)=$ $37,202 ; p<0,001)$.

\subsubsection{Influência da sustentabilidade sobre a intenção de compra}

Uma ANOVA foi realizada para verificar o efeito da sustentabilidade sobre a intenção de compra. Participantes demonstraram mais intenção de compra na condição com sustentabilidade $(M=4,03)$ do que na condição sem sustentabilidade $(M=3,39)$. Contudo, essa diferença não foi significativa entre os grupos $(F(1,62)=2,727 ; p=0,104)$. Esse resultado não oferece suporte à Hipótese 1a.

Neste contexto, ressalta-se que não foi possível comprovar que a inovação sustentável influencia positivamente a intenção de compra do consumidor, o que refuta os achados das pesquisas de Claudy, Peterson e O'Driscoll (2013) eClaudy, Garcia e O’Driscoll (2015), os quais destacam que atitudes positivas influenciam os consumidores a adotarem inovações ambientalmente sustentáveis.

Noppers et al. (2014) ressaltam que avaliações negativas de atributos ambientais influenciam a adoção de inovações sustentáveis, entretanto, para este grupo de respondentes do Estudo 1, as inovações sustentáveis não influenciam a sua intenção de compra, ou seja, o consumo sustentável não é fator relevante para os respondentes, o que também não está de acordo com os pressupostos teóricos de Bloemer e Ruyter (2002), os quais destacam que a tendência de mercado é que os consumidores aumentem a sua consciência ambiental.

\subsubsection{Influência da sustentabilidade sobre a disposição a pagar}

Para verificar o efeito da sustentabilidade sobre a disposição a pagar, foi realizada uma ANCOVA, tendo renda como uma variável de controle. Da mesma forma que na intenção de compra, a informação de sustentabilidade não teve influência significativa sobre a disposição a pagar. Os participantes que leram o cenário com sustentabilidade estavam dispostos a pagar, em média, $\mathrm{R} \$ 66,06$ pelo produto, enquanto os participantes que leram o cenário sem 
sustentabilidade estavam dispostos a pagar, em média, $\mathrm{R} \$ 72,90$ pelo produto. Embora a disposição a pagar tenha sido maior na condição sem sustentabilidade, essa diferença não foi significante $(\mathrm{F}(1,61)=1,681 ; p=0,200)$. Este resultado não oferece suporte à Hipótese $1 \mathrm{~b}$.

\subsubsection{Discussão}

Neste caso, a informação sobre a sustentabilidade ambiental não influenciou significativamente a intenção de compra e a disposição a pagar. Nesse sentido, foi realizado o estudo 2, para verificar se a variável luxo poderia modificar essa relação entre sustentabilidade ambiental e a intenção de compra e disposição a pagar.

Destaca-se que os novos segmentos de mercados e clientes preconizados por Hansen, Grosse-Dunker e Reichwald (2009), devem ser analisados, levando em conta as atitudes e percepções dos consumidores no que tange a sustentabilidade ambiental, no âmbito do consumo sustentável. Neste contexto, trata-se de um grande desafio para as organizações contemporâneas, o que segundo Dangelico e Devacshish (2010), poderá trazer o sucesso comercial de produtos ecologicamente corretos.

\subsection{ESTUDO 2}

\subsubsection{Verificaçãode manipulação}

Averificaçãode manipulação da sustentabilidade demonstrou que a manipulação foi bem sucedida, percebendo mais sustentabilidade na condição com sustentabilidade $(M=5,78)$ em comparação à condição sem sustentabilidade $(\mathrm{M}=3,00 ; \mathrm{F}(1,106)=90,753 ; p<0,001)$. Contudo, para a variável luxo, a diferença nas médias entre as condições com luxo $(\mathrm{M}=3,89)$ e sem luxo $(\mathrm{M}=3,40)$ não foi significante $(\mathrm{F}(1,106)=2,310 ; p=0,136)$.

\subsubsection{Influência do Luxo e da sustentabilidade sobre a intenção de compra}

Para verificar a influência da sustentabilidade e do luxo sobre a intenção de compra foi realizada uma ANOVA, onde não foi encontrado efeito de interação significante entre as duas variáveis independentes sobre intenção de compra $(\mathrm{F}(1,103)=0,081 ; p=0,777)$. Também não foi encontrado efeito principal significativo da sustentabilidade sobre intenção de compra (F $(1,103)=0,288 ; p=0,536$. Assim como no primeiro estudo, este resultado não oferece suporte à Hipótese 1a. Contudo, a variável luxo teve uma influência negativa significante 
sobre intenção de compra $(\mathrm{F}(1,103)=5,929 ; p<0,05)$, ou seja, independentemente do nível de sustentabilidade do produto descrito na situação, pessoas expostas à condição com luxo demonstraram menos intenção de compra do que as expostas na condição sem luxo (Figura 1). Esse resultado oferece suporte à Hipótese 2a.

Neste contexto, o efeito da informação da sustentabilidade do produto de luxo exerce um efeito negativo sobre intenção de compra do consumidor. Entretanto, as pesquisas de Fionda e Moore (2009) e Jin (2012), ressaltam que a elevada qualidade do material e a exclusividade são primordiais características do produto de luxo, impactariam a intenção de compra do consumidor. Contudo, Brun e Castelli (2013) destacam que o consumo simbólico, bem como as marcas de luxo, vem mantendo seus consumidores, o que não foi encontrado nesta pesquisa.

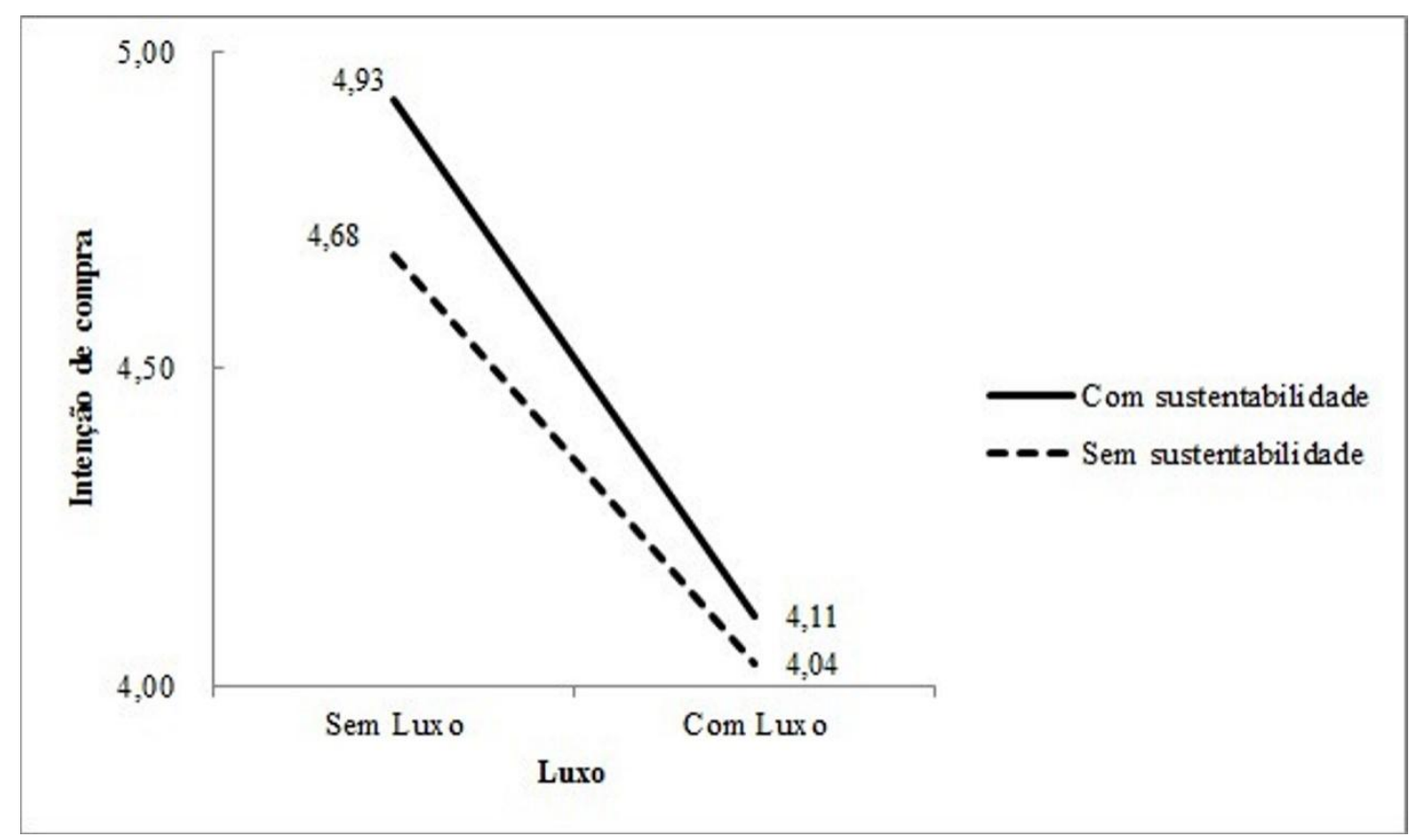

Figura 1 - Efeito do luxo e da sustentabilidade ambiental sobre a intenção de compra do consumidor Fonte: Dados da pesquisa (2015)

\subsubsection{Influência do Luxo e da sustentabilidade sobre a disposição a pagar}

Para verificar a influência da sustentabilidade e do luxo sobre a disposição a pagar foi realizada uma ANCOVA, tendo consumo por status e renda como covariáveis.Da mesma forma como na intenção de compra, não foi encontrado efeito de interação significante das duas variáveis independentes sobre a disposição a pagar $(\mathrm{F}(1,98)=0,465 ; p=0,497)$. Também, não foi encontrado efeito principal da sustentabilidade ambiental sobre a disposição a pagar (F $(1,98)=0,2,921 ; p=0,091)$, não oferecendo suporte à Hipótese $1 \mathrm{~b}$. Contudo, enquanto o luxo 
obteve um efeito principal negativo sobre a intenção de compra, conforme mencionado anteriormente, o seu efeito sobre a disposição a pagar foi positivo e significante $(\mathrm{F}(1,98)$ $=7,285 ; p<001$ ) (Figura 2), resultado que oferece suporte à Hipótese $2 b$.

Coerentemente, destaca-se o efeito da informação relacionada à sustentabilidade do produto de luxo exerce efeito positivo sobre a disposição a pagar, o que é corroborado nas pesquisas de Scarabotto et al. (2006), os quais enfatizam que o consumo de produtos de luxo envolve sentidos, ativando as emoções e as fantasias, os quais fortalecem a disposição a pagar.

Estes resultados ressaltam que consumidores de produtos de luxo estão dispostos a pagar mais por este tipo de produto, o que é resultante do prazer hedônico e da percepção utilitária deste produto (BODUR; GAO; GROHMANN, 2014; PATRICK, PROKOPEC, 2015).

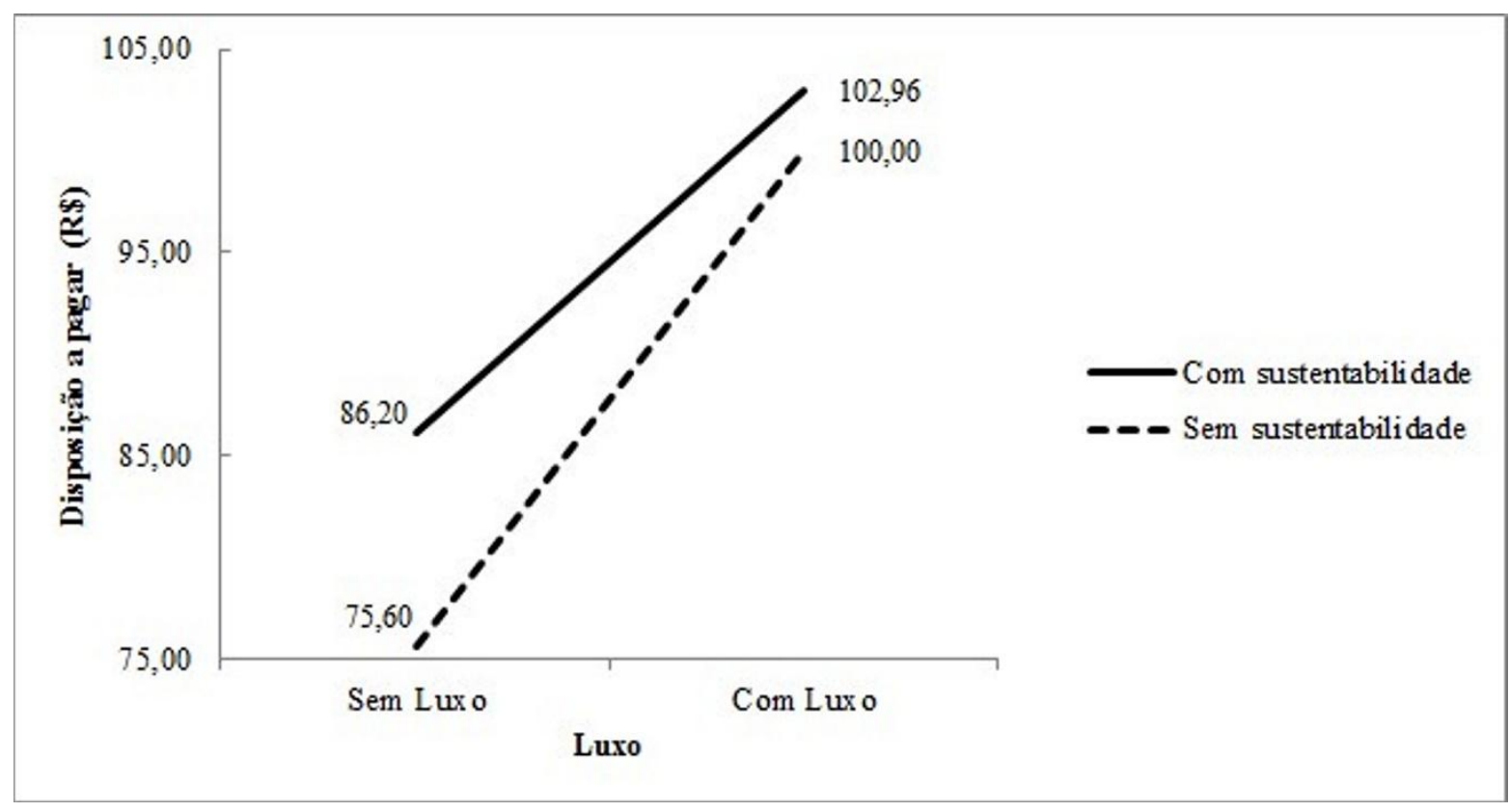

Figura 2 - Efeito do luxo e da sustentabilidade ambiental sobre a disposição a pagar do consumidor Fonte: dados da pesquisa (2015)

\section{CONSIDERAÇÕES FINAIS}

O objetivo desta pesquisa foi verificar a influência da inovação sustentável e do luxo sobre a intenção de compra do consumidor. Os principais resultados demonstraram que não foram encontrados efeitos significantes de interação entre as duas variáveis independentes manipuladas, a sustentabilidade e o luxo, sobre a intenção de compra e a disposição do consumidor a pagar pelo produto. Assim como, não foi encontrado efeito da sustentabilidade 
ambiental sobre a intenção de compra e da disposição a pagar. Por sua vez, o luxo teve um efeito negativo sobre a intenção de compra, mas positivo sobre a disposição a pagar.

Esse resultado corrobora com os argumentos de Almeida (2007), de que um dos desafios para o sucesso das inovações sustentáveis trata-se da resistência e falta de conhecimento da sociedade, em relação às soluções mais sustentáveis incorporadas aos produtos e serviços existentes no mercado. Dessa forma, essas açõespodem fazer com consumidores não se sintammotivados a consumir produtos mais sustentáveis, o que explica os efeitos não significantes nos estudos 1 e 2 .

Contudo, diante da crescente preocupação com os problemas ambientais, reduzir os impactos ambientais advindos da produção dos bens de consumo continua sendo relevante. Dessa forma, mesmo não havendo efeito significante da sustentabilidade na intenção de compra e disposição a pagar, o desenvolvimento de produtos ecologicamente corretos não deve ser uma prática abandonada pelas empresas, já que estes causam menos agressões ao meio ambiente (KLIMOVA; ZITEK, 2011).

No que tange às implicações acadêmicas e gerenciais, os achados da pesquisa permitiram o teste e a validação de métricas para a análise da influência da sustentabilidade e do luxo sobre a intenção de compra e da disposição apagar, corroborando para a academia, poisnecessita-se de meios para monitorar a intenção de compra dos consumidores. Neste contexto, a comunidade acadêmica, a sociedade e os profissionais das áreas afins terão métricas ligadas aos preceitos financeiros, pois muitas empresas no Brasil, sobretudo as de pequeno e médio porte, não realizampesquisas de consumo.

Ao longo da pesquisa, apresentaram-se algumas limitações, destacando-se principalmente no que se refere à sua capacidade de generalização, visto que foi explorada a realidade de 172 respondentes, divididos em dois gruposexperimentais. Como limitação do estudo, é importante ressaltar a não possibilidade de usar uma técnica de amostragem probabilística, de forma que a amostra fosse totalmente representativa da população. Outra limitação é manipulação da variável luxo, que apesar de ter um efeito significante, não teve médias estatisticamente diferentes de checagem entre os cenários, o que demonstra que pesquisas futuras podem manipular essa variável de diferentes maneiras. Além disso, destaca-se que as atitudes de um consumidor em relação a um produto ou uma marca não são apenas formadas instantaneamente, por meio de apenas uma fonte de informação, mas são também formadas ao longo do tempo através de diversas fontes de informação.

Por conseguinte, sugere-se uma análise mais abrangente, avaliando a influência da sustentabilidade e do luxo sobre a intenção de compra, assim como a disposição apagar, a um

REAd | Porto Alegre - Edição 86 - No 2 - Maio / Agosto 2017 - p. 258 - 273 
número maior de respondentes. Em acréscimo, são pertinentes estudos que enfoquem a comparação entre regiões, setores, cadeias produtivas ou até mesmo as diferentes percepções entre diversos atores inseridos neste contexto.Assim, pesquisas futuras podem utilizar diferentes métodos - como surveys - para verificar as razões pelas quais consumidores adotam as inovações sustentáveis.

Neste artigo, foi encontrada a informação de que a sustentabilidade de um produto não obteve efeito sobre a intenção de compra e a disposição a pagar do consumidor. Contudo, é importante ressaltar que nas condições sem a informação sobre a sustentabilidade, em ambos os experimentos, também não havia informação que o produto causava danos ao meio ambiente. Entretanto, é possível que uma informação negativa sobre a sustentabilidade forme atitudes contrárias do consumidor em relação ao produto, influenciando negativamente a intenção de compra. Em outras palavras, é possível que estas informações sobre a sustentabilidade tenham uma influência maior na formação de atitudes negativas do consumidor do que a influência das informações positivas na formação de atitudes também positivas. Estudos futuros poderiam investigar esse aspecto.

Além disso, outras medidas para verificar o possível sucesso de inovações sustentáveis, como interesse e aceitabilidade, que podem ser atitudes precedentes à intenção de compra.

\section{REFERÊNCIAS}

ALMEIDA, F. Os desafios da sustentabilidade. Rio de Janeiro: Elsevier, 2007.

BERRY, C. J. The Idea of Luxury: a conceptual and historical investigation. Cambridge: Cambridge University Press, 1994.

BLOEMER, J., RUYTER, K. The impact of attitudes strength on the acceptance of green services. Journal of Retailing and Consumer Services, Amsterdam, v. 9, n. 1, p. 45-52, 2002.

COOPER, R. G. New product: The Factors That Drive Sucess. International Marketing Review. Bingley, v. 11, n. 1, p. 60-76, 1994.

CLAUDY, M. C.; GARCIA, R.; O’DRISCOLL, A. Consumer resistance to innovation: a behavioral reasoning perspective. Journal of the Academy of Marketing Science, New York, v. 43, n. 4, p. 528-544, 2015.

CLAUDY, M. C.; PETERSON, M.; O'DRISCOLL, A. Understanding the attitude-behavior gap for renewable energy systems using behavioral reasoning theory. Journal of

Macromarketing, Thousand Oaks, v. 33, n. 5, p. 273-287, 2013.

REAd | Porto Alegre - Edição 86 - No 2 - Maio / Agosto 2017 - p. 258 - 273 
DANGELICO, R. M.; DEVACSHISH, P. Mainstreaming Green Product Innovation: Why and How Companies Integrate Environmental Sustainability. Journal of Business Ethics, New York, v. 95, n. 3, p. 471-486, 2010.

D`SOUZA, C.; TAGHIAN, M.; KHOSLA, R. Examination of environmental beliefs and its impact on the influence of price, quality and demographic characteristics with respect to green purchase intention. Journal of Targeting, Measurement andAnalysis for Marketing, New York, v. 15, n. 2, p. 69-78, 2007.

FULLER, D. A. Sustainable Marketing. Thousand Oaks, CA: Sage Publications, 1999.

GUIMARÃES, J. C. F. A relação entre recursos estratégicos e inovação de produto para a obtenção de vantagem competitiva em empresas da indústria moveleira. 2013, 144 f.Tese (Doutorado em Administração) - Programa de Pós-Graduação em Administração, Universidade de Caxias do Sul - UCS e Pontifícia Universidade Católica do Rio Grande do Sul - PUC/RS, Caxias do Sul, 2013.

HANSEN, E.; GROSSE-DINKER, F.; REICHWALD, R. Sustainability innovation cube: a framework to evaluate sustainability oriented innovations. International Journal of Innovation Management, [s.1.], v. 13, n. 4, p. 683-713, 2009.

HEINE, K.; PHAN, M. Trade up Mass-Market Goods to Luxury Products. Australian Marketing Journal, Amsterdam, v. 19, n. 2, 2011.

HOMBURG, C.; KOSCHATE, N.; HOYER, W. D. Do satisfied customers really pay more? A study of the relationship between customer satisfaction and willingness to pay. Journal of Marketing, Chicago, v. 69, n. 2, p. 84-96, 2005.

KLEINSCHMIDT, E. J.; COOPER, R. G. The impact of product innovatiness on performance. Journal of Product Innovation Management, Hoboken, v. 8, n. 4, p. 240$251,1991$.

KLÍMOVÁ, V.; ZITEK, V. Eco-innovation as a result companies innovation activities. ELeader online, 2011. Disponível em: <http://www.gcasa.com/conferences/zagreb/papers/Klimova_Zitek.pdf>. Acesso em: 12 nov. 2015.

LAGES, N. S; NETO, A. V. Mensurando a consciência ecológica do consumidor: um estudo realizado na cidade de Porto Alegre. In: ENCONTRO ANUAL DA ASSOCIAÇÃO

NACIONAL DE PÓS-GRADUAÇÃO EM ADMINISTRAÇÃO, 26., Salvador, 2002. Anais...Salvador: ANPAD 2002.

McCRACKEN, G. Culture and consumption: A theoretical account of the structure and movement of the cultural meaning of consumer goods. Journal of Consumer Research, [s.l.], v. 13, n. 1, p. 71-84, 1986.

NOPPERS, E. H. et al. The adoption of sustainable innovations: driven by symbolic and environmental motives. Global Environmental Change, Amsterdam, v. 25, p. 52-62, 2014. 
OCDE. Manual de Oslo: diretrizes para coleta e interpretação de dados sobre inovação. 3. ed. Rio de Janeiro: Finep, 2005.

OTTMAN, J. A. Marketing Verde: desafios e oportunidades para a nova era do marketing. São Paulo: Makron Books, 1994.

SCARABOTTO, D. et al. Pequenos Luxos, Grandes Prazeres: Significado do Consumo e Valores dos Consumidores de Joalheria e Vestuário de Luxo. In: ENCONTRO DE MARKETING DA ANPAD, 2., Rio de Janeiro, 2006. Anais... Rio de Janeiro: ANPAD, 2006.

SCHUMPETER, J. A. Capitalismo, socialismo e democracia. Rio de Janeiro: Fundo de Cultura, 1961.

SEVERO, E. A. A. Inovação e sustentabilidade ambiental nas empresas do Arranjo Produtivo Local Metalmecânico Automotivo da Serra Gaúcha. 234 p. Tese (Doutorado em Administração) - Programa de Pós-Graduação em Administração, Universidade de Caxias do Sul - UCS, Caxias do Sul, 2013.

STREHLAU, S.; ARANHA, F. Adaptação e Validação da Escala de Consumo de Status (SCS) para uso no contexto brasileiro. Revista de Administração Faces Journal, Belo Horizonte, v. 3, n. 1, p. 9-17, 2004.

SOMBART, W. Luxury and Capitalism. Ann Arbor: University of Michigan Press, 1967.

TYNAN, C.; McKECHNIE, S.; CHUON, C. Co-creating value for luxury brands. Journal of Business Research, Amstedam, v. 63, n. 11, p. 1156-1163, 2010. 